\title{
Hydrophilic Derivatives of Dithranol
}

\author{
Helene Tanzer, Matthias Seidel, and Wolfgang Wiegrebe***) \\ Institute of Pharmacy, University, P.O. Box 397, D-8400 Regensburg
}

Received December 22, 1987

The syntheses of dithranol derivatives with $\omega$-carboxyalkyl side chains at C-2 (and C-7) or $\omega$-methoxycarbonylacyl-substituents at C-10, respectively, are described.

\section{Hydrophile Dithranolderivate}

Die Herstellung von Dithranolderivaten mit $\omega$-Carboxyalkyl-Seitenketten an C-2 (und C-7) bzw. mit $\omega$-Methoxycarbonylacyl-Substituenten an $\mathrm{C}-10$ wird beschrieben.
We have reported a) upon the inhibition of glucose-6-phosphate-dehydrogenase by dithranol (1) ${ }^{1)}$, b) upon the UV-spectra of 1 and its anion ${ }^{2)}$ and c) upon the active oxygen species generated by 1-C-10-anion ${ }^{3)}$. These experiments were hampered by the low degree of solubility of $\mathbf{1}$ in aqueous buffer systems.

Here we describe the syntheses of hydrophilic derivatives of 1 which will be used as model compounds for further experiments in the context of the problems a)-c) mentioned above.

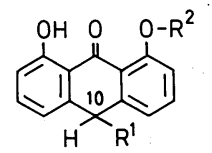

$$
\begin{aligned}
& \text { 1: } R^{1}=R^{2}=H \\
& \text { 2: } R^{1}=n-C O-C_{3} H_{7}, R^{2}=H \\
& \text { 3: } R^{1}=C O-\left(\mathrm{CH}_{2}\right)_{2}-\mathrm{COOCH}_{3}, R^{2}=\mathrm{H} \\
& \text { 4: } R^{1}=\mathrm{CO}-\left(\mathrm{CH}_{2}\right)_{3}-\mathrm{COOCH}_{3}, R^{2}=\mathrm{H} \\
& \text { 5: } R^{1}=\mathrm{H}, R^{2}=\mathrm{CO}-\left(\mathrm{CH}_{2}\right)_{3}-\mathrm{COOCH}_{3}
\end{aligned}
$$<smiles>O=C1CCC(=C2c3cccc(O)c3C(=O)c3c(O)cccc32)O1</smiles>

\section{A) C-10-Acylated Dithranol Derivatives}

C-10-acylated derivatives of 1 have been investigated thoroughly by Mustakallio et al. ${ }^{4)}$ mainly under clinical aspects, butantrone (2) being the most potent compound of this series. Unfortunately $\mathbf{2}$ is not suitable for our purposes. So we converted 1 into the esters 3 and 4, respectively, by acylation with $\omega$-methoxycarbonyl-propionyl chloride ${ }^{5)}$ or the homologous $\omega$-methoxycarbonyl-butyryl chloride.

Besides the main components 3 and 4 the phenyl ester 5 arose as a side product. - 1 reacted with succinyl chloride yielding the lactone 6.<smiles>[R]c1ccc2c(c1O)C(=O)c1c(ccc([R])c1O)C2</smiles><smiles>[R]Oc1c([R])ccc2c1C(=O)c1ccc([R])c(O)c1C2=O</smiles>

7: $\mathrm{R}^{1}=\mathrm{CH}_{2}-\mathrm{CH}_{2}-\mathrm{COOH}, \mathrm{R}^{2}=\mathrm{H}$

12: $R^{1}=R^{2}=\mathrm{CH}_{2}-\mathrm{COOH}$

15: $\mathrm{R}^{1}=\mathrm{CH}_{2}-\mathrm{COOH}, \mathrm{R}^{2}=\mathrm{H}$

$$
\begin{aligned}
& \text { 8: } R^{2}=\mathrm{CH}_{2}-\mathrm{CH}\left(\mathrm{COOC}_{2} \mathrm{H}_{5}\right)_{2}, R^{4}=\mathrm{CH}_{3} \\
& R^{1}=R^{3}=\mathrm{H} \\
& \text { 9: } R^{1}-R^{4}=\mathrm{H} \\
& \text { 10: } R^{2}=R^{3}=\mathrm{CH}_{2}-\mathrm{COOH}, R^{1}=R^{4}=\mathrm{H} \\
& \text { 11: } R^{2}=R^{3}=\mathrm{CH}_{2}-\mathrm{COOCH}_{3}, R^{1}=R^{4}=\mathrm{CH}_{3} \\
& \text { 13: } R^{4}=\mathrm{CH}_{3}, R^{1}-R^{3}=\mathrm{H} \\
& \text { 14: } R^{2}=\mathrm{CH}_{2}-\mathrm{COOH}, \mathrm{R}^{4}=\mathrm{CH}_{3}, \mathrm{R}^{1}=\mathrm{R}^{3}=\mathrm{H}
\end{aligned}
$$

\footnotetext{
${ }^{* *}$ ) Herrn Prof. Dr. Zymalkowski, Bonn, in Verehrung zum 75. Geburtstag gewidmet.
}

\section{B) C-2 (and C-7) Substituted Dithranol Derivatives}

2-(2-carboxyethyl)-dithranol 7 was obtained by ether cleavage, saponification, decarboxylation, and reduction of the anthraquinone $8^{6)}$ with $\mathrm{SnCl}_{2}$ in a one-pot-reaction according to Auterhoff ${ }^{7}$.

Chrysazin (9) when being treated with an excess of $(\mathrm{HO})_{2}$ $\mathrm{CH}-\mathrm{COOH} / \mathrm{NaOH} / \mathrm{Na}$-dithionite according to Marschalk $^{8)}$ yielded the bis-(carboxymethyl)-dihydroxyanthraquinone 10 as a very polar, crude material which was methylated to $\mathbf{1 1}$ for characterization. Under Auterhoff's reductive conditions ${ }^{7)} 11$ was converted to 2,7-bis-(carboxymethyl)-dithranol 12.

Analogously to the reaction of chrysazin (9) its mono-methyl ether $13^{6)}$ was converted with $(\mathrm{HO})_{2} \mathrm{CH}-\mathrm{COOH}$ to the mono-carboxymethyl-derivative 14 , which was reduced to the carboxymethyldithranol $\mathbf{1 5}$ with concomittant ether cleavage.

\section{Experimental Part}

Devices: Mp.: (uncorr.) apparatus according to Dr. Tottoli (Büchi). - UVspectra: Shimadzu 210; $1 \mathrm{~cm}$ cells. - IR-spectra in $\mathrm{KBr}$ : Beckman Acculab III. - ${ }^{1} \mathrm{H}$-NMR-spectra: Varian EM 390 (90 MHz), $\mathrm{CDCl}_{3}, 35^{\circ} \mathrm{C}$, TMS as int. stand. - MS: Varian MAT CH5, 70 eV. - NI-FAB-MS (glycerol/DMSO 1:1; Xe) Varian MAT 311A. - All the reactions were performed under $\mathrm{N}_{2}$ and light protection.

\section{Section A}

1,8-Dihydroxy-10-(3'-methoxycarbonyl-1'-oxopropyl)-9(10H)anthracenone (3)

The suspension of $2.26 \mathrm{~g}(10 \mathrm{mmol}) 1,1.76 \mathrm{~g}(11.7 \mathrm{mmol}) 3$-methoxycarbonyl-propionyl chloride ${ }^{5)}$ and $1.07 \mathrm{~g}(13.3 \mathrm{mmol})$ of dry pyridine in $70 \mathrm{ml}$ of absol. toluene was refluxed for $4 \mathrm{~h}$. - The resulting orange solution was evaporated i. vac., the residue, dissolved in a small volume of $\mathrm{CH}_{2} \mathrm{Cl}_{2}$, was purified by $\mathrm{CC}\left(\mathrm{SiO}_{2} / \mathrm{Et}_{2} \mathrm{O}\right)$ and crystallization from $\mathrm{Et}_{2} \mathrm{O}: 1.4 \mathrm{~g}(41 \%) 3$, yellow crystals, m. p. $122^{\circ} \mathrm{C} .-\mathrm{rf}\left(\mathrm{SiO}_{2} ; \mathrm{Et}_{2} \mathrm{O}\right): 0.74 .-\mathrm{C}_{19} \mathrm{H}_{16} \mathrm{O}_{6}(340.3)$ Calc. C $67.1 \mathrm{H} 4.71$ Found C $66.9 \mathrm{H}$ 4.74. - UV $\left(\mathrm{CH}_{2} \mathrm{Cl}_{2}\right): \lambda \max (\log \varepsilon)$ $=357$ (4.01), 279 (4.00), 267 (4.02), $237 \mathrm{~nm}$ (3.80). - IR: 1745 (CO$\left.\mathrm{OCH}_{3}\right), 1725(\mathrm{C}=\mathrm{O}), 1635 \mathrm{~cm}^{-1}(\mathrm{C}=\mathrm{O} \cdots \mathrm{HO}) .-{ }^{1} \mathrm{H}-\mathrm{NMR}: \delta(\mathrm{ppm})=$ $12.0(\mathrm{~s} ; 2 \mathrm{OH}), 7.70-6.82(\mathrm{~m} ; 6 \mathrm{H}$ aromat. $), 5.29(\mathrm{~s} ; 1 \mathrm{H}$ at $\mathrm{C}-10), 3.61(\mathrm{~s}$; $\left.3 \mathrm{H}, \mathrm{CH}_{3}\right), 2.42\left(\mathrm{~s} ; 4 \mathrm{H}, 2 \times \mathrm{CH}_{2}\right)$. 


\section{1,8-Dihydroxy-10-(4'-methoxycarbonyl-1'-oxobutyl)-9(10H)anthrace- none (4)}

From $2.26 \mathrm{~g} \mathrm{(10} \mathrm{mmol)} \mathrm{1,} 1.92 \mathrm{~g}(11.7 \mathrm{mmol})$ 4-methoxycarbonyl-butyryl chloride (Aldrich Chemicals) and $1.07 \mathrm{~g}(13.3 \mathrm{mmol})$ of dry pyridine in toluene as described for 3: $1.8 \mathrm{~g}(50 \%)$ yellow crystals from $\mathrm{Et}_{2} \mathrm{O} /$ petrol ether $\left(40-60^{\circ} \mathrm{C}\right)(1: 1)$, m.p. $92^{\circ} \mathrm{C} .-\mathrm{rf}\left(\mathrm{SiO}_{2} ; \mathrm{Et}_{2} \mathrm{O} /\right.$ petrol ether 1:1): 0.48. $-\mathrm{C}_{20} \mathrm{H}_{18} \mathrm{O}_{6}$ (354.4) Calc. C $67.8 \mathrm{H} \mathrm{5.08}$ Found C $67.8 \mathrm{H} \mathrm{5.10.-}$ $\mathrm{UV}\left(\mathrm{CH}_{2} \mathrm{Cl}_{2}\right): \lambda \max (\log \varepsilon)=356(4.04), 280(4.02), 268(4.02), 233 \mathrm{~nm}$ (3.92). - IR: $1735\left(\mathrm{CO}_{-} \mathrm{OCH}_{3}\right), 1715(\mathrm{C}=\mathrm{O}), 1635 \mathrm{~cm}^{-1}(\mathrm{C}=\mathrm{O} \cdots \mathrm{HO})$. ${ }^{1} \mathrm{H}-\mathrm{NMR}: \delta(\mathrm{ppm})=11.82(\mathrm{~s} ; 2 \mathrm{OH}), 7.61-6.82(\mathrm{~m} ; 6 \mathrm{H}$ aromat.), $5.21(\mathrm{~s}$; $1 \mathrm{H}$ at $\mathrm{C}-10), 3.53\left(\mathrm{~s} ; 3 \mathrm{H}, \mathrm{CH}_{3}\right), 2.19-1.96\left(\mathrm{~m} ; 4 \mathrm{H}, \mathrm{CH}_{2}\right.$ at $\mathrm{C}-2^{\prime}$ and $\mathrm{CH}_{2}$ at $\left.\mathrm{C}-4^{\prime}\right), 1.77-1.54\left(\mathrm{~m} ; 2 \mathrm{H}, \mathrm{CH}_{2}\right.$ at $\left.\mathrm{C}-3^{\prime}\right)$.

\section{1-(4'-Methoxycarbonyl-butyryloxy)-8-hydroxy-9(10H)-anthracenone (5)}

Fractional crystallization of the mother liquors of 4 from $\mathrm{Et}_{2} \mathrm{O} /$ petrol ether (1:1) afforded $110 \mathrm{mg} \mathrm{(3.1 \% )} 5$ as yellow crystals, m.p. $103^{\circ} \mathrm{C} .-\mathrm{rf}$ $\left(\mathrm{SiO}_{2} ; \mathrm{Et}_{2} \mathrm{O}\right.$ /petrol ether 1:1): 0.42. $-\mathrm{C}_{20} \mathrm{H}_{18} \mathrm{O}_{6}$ (354.4). Calc. C 67.8 H 5.08 Found C $67.9 \mathrm{H} 5.09$. - UV $\left(\mathrm{CH}_{2} \mathrm{Cl}_{2}\right): \lambda \max (\log \varepsilon)=357(3.67)$, 281 (4.12), 255 (4.09), 233 (3.93), 220 nm (3.47). - IR: 1765 (O-CO-R), $1735\left(\mathrm{CO}-\mathrm{OCH}_{3}\right), 1640 \mathrm{~cm}^{-1}(\mathrm{C}=\mathrm{O} \cdots \mathrm{H}-\mathrm{O}) .-{ }^{1} \mathrm{H}-\mathrm{NMR}: \delta(\mathrm{ppm})=$ $12.22(\mathrm{~s} ; 1 \mathrm{OH}), 7.64-6.78(\mathrm{~m} ; 6 \mathrm{H}$ aromat.), 4.34 (s; $2 \mathrm{H}$ at $\mathrm{C}-10), 3.74(\mathrm{~s}$; $\left.3 \mathrm{H}, \mathrm{CH}_{3}\right), 2.88-2.71\left(\mathrm{~m} ; 2 \mathrm{H}, \mathrm{CH}_{2}\left(\mathrm{C}-2^{\prime}\right)\right), 2.61-2.47\left(\mathrm{~m} ; 2 \mathrm{H}, \mathrm{CH}_{2}\right.$ $\left.\left(\mathrm{C}-4^{\prime}\right)\right), 2.29-2.03\left(\mathrm{~m} ; 2 \mathrm{H}, \mathrm{CH}_{2}\left(\mathrm{C}-3^{\prime}\right)\right)$.

\section{1,8-Dihydroxy-10-(5'-oxo-tetrahydrofuryliden-2')-9(10H)anthracenone}

(6)

A suspension of $2.26 \mathrm{~g}(10 \mathrm{mmol}) \mathbf{1}$ in $40 \mathrm{ml}$ of absol. toluene and $1.07 \mathrm{~g}$ $(13.33 \mathrm{mmol})$ of dry pyridine was added to $1.6 \mathrm{~g}$ succinyl chloride (10 $\mathrm{mmol}$ ) in $40 \mathrm{ml}$ of absol. toluene. After refluxing for $3.5 \mathrm{~h}$ the orange solution thus formed was evaporated $\mathrm{i}$. vac. and the residue was dissolved in a small volume of $\mathrm{CH}_{2} \mathrm{Cl}_{2}$. $\mathrm{CC}\left(\mathrm{SiO}_{2} / \mathrm{CH}_{2} \mathrm{Cl}_{2}\right)$ and crystallization from $\mathrm{CH}_{2} \mathrm{Cl}_{2}$ afforded $1.1 \mathrm{~g}(36 \%) 6$ as yellow crystals, mp. $245^{\circ} \mathrm{C}$. $-\mathrm{rf}\left(\mathrm{SiO}_{2}\right.$; $\mathrm{CH}_{2} \mathrm{Cl}_{2}$ ): 0.64. $-\mathrm{C}_{18} \mathrm{H}_{12} \mathrm{O}_{5}$ (308.5) Calc. C 70.1 H 3.90 Found C 69.6 H 3.93. - UV $\left(\mathrm{CH}_{2} \mathrm{Cl}_{2}\right): \lambda \max (\log \varepsilon)=384$ (4.36), 303 (sh, 4.31), 293 (4.32), 242 (4.41), 220 (4.06), $210 \mathrm{~nm}$ (4.05). - IR: 1810 (CO-O), 1640 $\mathrm{cm}^{-1}(\mathrm{C}=\mathrm{O} \cdots \mathrm{HO}) .-{ }^{1} \mathrm{H}-\mathrm{NMR}\left(\left[\mathrm{D}_{6}\right] \mathrm{DMSO}\right): \delta(\mathrm{ppm})=11.38(\mathrm{~s} ; 1 \mathrm{OH})$, $11.30(\mathrm{~s} ; 1 \mathrm{OH}), 7.85-6.80\left(\mathrm{~m} ; 6 \mathrm{H}\right.$ aromat.) $3.56-3.32\left(\mathrm{~m} ; 2 \mathrm{H}, \mathrm{CH}_{2}\right.$ $\left.\left(\mathrm{C}-4^{\prime}\right)\right), 2.89-2.67\left(\mathrm{~m} ; 2 \mathrm{H}, \mathrm{CH}_{2}\left(\mathrm{C}-3^{\prime}\right)\right)$. $-\mathrm{MS}: \mathrm{m} / \mathrm{z}=309(20 \%), 308$ $\left(100 ; \mathrm{M}^{+\cdot}\right), 280$ (63), 263 (18), 253 (37), 252 (19), 238 (27), 224 (28).

\section{Section $B$}

\section{3-(1,8-Dihydroxy-9(10H)-anthracenon-2-yl)-propionic acid (7)}

$10 \mathrm{~g} \mathrm{SnCl}_{2}$ in $25 \mathrm{ml}$ of fuming $\mathrm{HCl}$ were added drop by drop to a solution of $1 \mathrm{~g}(2.3 \mathrm{mmol})$ (9,10-dihydro-1-hydroxy-8-methoxy-9,10-dioxo-2-anthryl)methyl malonic acid diethyl ester (8) $\left.{ }^{6}\right)$ in $50 \mathrm{ml}$ of boiling acetic acid (100\%). Boiling was continued for $6 \mathrm{~h}$. After cooling 7 was precipitated by addition of 3-4 ml of water: $490 \mathrm{mg}(70 \%) 7, \mathrm{mp} .>220^{\circ}$ (decomp.) from $\mathrm{CH}_{2} \mathrm{Cl}_{2} / \mathrm{MeOH}$. $-\mathrm{rf}\left(\mathrm{SiO}_{2} ; \mathrm{CH}_{2} \mathrm{Cl}_{2} / \mathrm{MeOH}\right.$ 9:1): 0.55. $-\mathrm{C}_{17} \mathrm{H}_{14} \mathrm{O}_{5}$ (298.3) Calc. C 68.4 H 4.70 Found C 68.2 H 4.65. - UV (MeOH): $\lambda$ max $(\log \varepsilon)=357(4.00), 291(4.06), 257$ (4.14), $207 \mathrm{~nm}(4.40)$. IR: 1710 $(\mathrm{COOH}), 1620 \mathrm{~cm}^{-1}(\mathrm{C}=\mathrm{O} \cdots \mathrm{HO}) .-{ }^{1} \mathrm{H}-\mathrm{NMR}\left(\mathrm{CF}_{3} \mathrm{COOD}\right): \delta(\mathrm{ppm})=$ 6.99-6.77 (m; 2H aromat.), 6.47-6.18 (m;3H aromat.), $3.55(\mathrm{~s} ; 2 \mathrm{H}$, $\left.\mathrm{CH}_{2}\right), 2.55-2.09$ (m; 4H, $\mathrm{CH}_{2} \mathrm{CH}_{2}$-CO). - NI-FAB-MS: $\mathrm{m} / \mathrm{z}=298$ $\left(38 \% ; \mathrm{M}^{-}\right), 297$ [100; (M-H) $], 253\left(19 ; 297-\mathrm{CO}_{2}\right)$.

\section{2,7-Bis-(carboxymethyl)-9,10-dihydro-1,8-dihydroxy-anthracene-9,10- dione (10)}

A mixture of $4 \mathrm{~g}(17 \mathrm{mmol}) 9$ and $13 \mathrm{~g}(176 \mathrm{mmol})$ glyoxylic acid hydrate (Janssen Chimica) in $250 \mathrm{ml} \mathrm{MeOH}$ and $150 \mathrm{ml} 2 \mathrm{~N} \mathrm{NaOH}$ was heated under $\mathrm{N}_{2}$ to $70^{\circ} \mathrm{C}$. Na-dithionite was added in portions at this temp. until the colour changed from red violet to brown (about $3 \mathrm{~h}$ ). Then the mixture was acidified with $2 \mathrm{~N} \mathrm{HCl}$, the precipitate was filtered by suction and dried: crude material of $\mathbf{1 0}$.
2,7-Bis-(methoxycarbonylmethyl)-9,10-dihydro-1,8-dimethoxy-anthracene-9,10-dione (11)

$1 \mathrm{~g}(2.8 \mathrm{mmol}) 10$ and $2.3 \mathrm{~g} \mathrm{~K}_{2} \mathrm{CO}_{3}$ were refluxed in $40 \mathrm{ml}$ dry acetone for $5 \mathrm{~h}$. During this period $1.2 \mathrm{ml}$ of dimethyl sulfate were added drop by drop. - After cooling and addition of $100 \mathrm{ml} 2 \mathrm{~N} \mathrm{HCl}$ the mixture was extracted with $\mathrm{CH}_{2} \mathrm{Cl}_{2}$, the org. phase was washed with water, dried over $\mathrm{Na}_{2} \mathrm{SO}_{4}$ and evaporated. The residue was purified by $\mathrm{CC}\left(\mathrm{SiO}_{2} ; \mathrm{CH}_{2} \mathrm{Cl}_{2}\right)$ : $500 \mathrm{mg}(43 \%) 11, \mathrm{mp} .124^{\circ} \mathrm{C}\left(\mathrm{CH}_{2} \mathrm{Cl}_{2} /\right.$ petrol ether $) .-\operatorname{rf}\left(\mathrm{SiO}_{2}\right.$; $\mathrm{CH}_{2} \mathrm{Cl}_{2}$ ): 0.32. $-\mathrm{C}_{22} \mathrm{H}_{20} \mathrm{O}_{8}(412.4)$ Calc. C $64.1 \mathrm{H} 4.85$ Found $\mathrm{C} 64.0$ $\mathrm{H} 4.85$. - UV (MeOH): $\lambda \max (\log \varepsilon)=356(3.87), 255(4.47), 218 \mathrm{~nm}$ (4.43). - IR: $1740\left(\mathrm{CO}^{-\mathrm{OCH}_{3}}\right), 1687 \mathrm{~cm}^{-1}(\mathrm{C}=\mathrm{O}) .-{ }^{1} \mathrm{H}-\mathrm{NMR}: \delta(\mathrm{ppm})=$ 8.09 (AB; $\left.2 \mathrm{H}, \mathrm{H}-4, \mathrm{H}-5, \mathrm{~J}_{\mathrm{AB}}=7 \mathrm{~Hz}\right), 7.69(\mathrm{AB} ; 2 \mathrm{H}, \mathrm{H}-3, \mathrm{H}-6), 4.03$ (s; $\left.6 \mathrm{H}, \mathrm{OCH}_{3}\right) 3.86\left(\mathrm{~s} ; 4 \mathrm{H}, \mathrm{CH}_{2}\right), 3.80\left(\mathrm{~s} ; 6 \mathrm{H}, \mathrm{CO}-\mathrm{OCH}_{3}\right)$.

\section{2,7-Bis-(carboxymethyl)-1,8-dihydroxy-9(10H)-anthracenone (12)}

$100 \mathrm{mg}(0.24 \mathrm{mmol}) 11$ in $15 \mathrm{ml}$ glacial acetic acid were reduced with $1.25 \mathrm{~g} \mathrm{SnCl}_{2}$ in $3 \mathrm{ml}$ of fuming $\mathrm{HCl}$ as described for 7. - After cooling 12 crystallized from the mixture in a pure state: $40 \mathrm{mg} \mathrm{(48 \% )} \mathrm{12,} \mathrm{mp.} \mathrm{>}$ $250{ }^{\circ} \mathrm{C}$ (decomp.) from acetic acid. $-\mathrm{C}_{18} \mathrm{H}_{14} \mathrm{O}_{7}$ (342.3) Calc. C63.2 H 4.09 Found C $63.0 \mathrm{H} 4.14$. - UV $(\mathrm{MeOH}): \lambda \max (\log \varepsilon)=361(3.98)$, 293 (4.02), $260 \mathrm{~nm}$ (4.23). - IR: $1700(\mathrm{COOH}), 1620 \mathrm{~cm}^{-1}(\mathrm{C}=\mathrm{O} \cdots \mathrm{HO})$, - ${ }^{1} \mathrm{H}-\mathrm{NMR}\left(60 \mathrm{MHz},\left[\mathrm{D}_{6}\right] \mathrm{DMSO}\right): \delta(\mathrm{ppm})=12.41(\mathrm{~s} ; 2 \mathrm{H}, \mathrm{OH}), 7.58$ $\left(\mathrm{AB} ; 2 \mathrm{H}, \mathrm{J}_{\mathrm{AB}}=10 \mathrm{~Hz}\right), 7.02(\mathrm{AB} ; 2 \mathrm{H}), 4.42\left(\mathrm{~s} ; 2 \mathrm{H}, \mathrm{CH}_{2}\right), 3.62(\mathrm{~s} ; 4 \mathrm{H}$, $\mathrm{CH}_{2}$-CO). - NI-FAB-MS: $\mathrm{m} / \mathrm{z}=342\left(35 \% ; \mathrm{M}^{--}\right), 341$ [100; $\left.(\mathrm{M}-\mathrm{H})^{-}\right]$, $297\left(21 ; 341-\mathrm{CO}_{2}\right), 253\left(95 ; 297-\mathrm{CO}_{2}\right)$.

\section{2-Carboxymethyl-9,10-dihydro-1-hydroxy-8-methoxy-anthracene-9,10-} dione (14)

To $500 \mathrm{mg}(1.9 \mathrm{mmol}) 13$ and $1.5 \mathrm{~g}$ glyoxylic acid hydrate in $100 \mathrm{ml}$ $\mathrm{MeOH}$ and $50 \mathrm{ml} 2 \mathrm{~N} \mathrm{NaOH}$ was added an excess of $\mathrm{Na}$-dithionite at $60{ }^{\circ} \mathrm{C}$ under $\mathrm{N}_{2}$. After heating for $2 \mathrm{~h}$ the mixture was acidified with 100 $\mathrm{ml} 2 \mathrm{~N} \mathrm{HCl}$ and extracted with $\mathrm{CH}_{2} \mathrm{Cl}_{2}$. After evaporation to a small volume this solution was purified bei $\mathrm{CC}\left(\mathrm{SiO}_{2} ; \mathrm{CH}_{2} \mathrm{Cl}_{2} / \mathrm{MeOH} 100: 14 \mathrm{v} /\right.$ v): $220 \mathrm{mg}$ (36\%) 14, mp. $>258^{\circ} \mathrm{C}$ (decomp.) (acetic acid/water 9:1). - rf $\left(\mathrm{SiO}_{2} ; \mathrm{CH}_{2} \mathrm{Cl}_{2} / \mathrm{MeOH}\right.$ 9:1): 0.41. $-\mathrm{C}_{17} \mathrm{H}_{12} \mathrm{O}_{6}+1 / 2 \mathrm{H}_{2} \mathrm{O}$ (321.3) Calc. C $63.6 \mathrm{H} 4.05$ Found C $63.8 \mathrm{H}$ 3.90. - UV (MeOH): $\lambda \max (\log \varepsilon)=416$ (3.98), 258 (4.51), $225 \mathrm{~nm}$ (4.52). - IR: $1690(\mathrm{COOH}), 1665(\mathrm{C}=\mathrm{O}), 1630$ $\mathrm{cm}^{-1}(\mathrm{C}=\mathrm{O} \cdots \mathrm{HO}) .{ }^{1} \mathrm{H}-\mathrm{NMR}\left(\mathrm{CF}_{3} \mathrm{COOH}\right): \delta(\mathrm{ppm})=7.55-6.97(\mathrm{~m}$; $5 \mathrm{H}$ aromat.), $3.53\left(\mathrm{~s} ; 3 \mathrm{H}, \mathrm{OCH}_{3}\right), 3.41\left(\mathrm{~s} ; 2 \mathrm{H}, \mathrm{CH}_{2} \mathrm{CO}\right)$.

\section{(1,8-Dihydroxy-9(10H)-anthracenon-2-yl)acetic acid (15)}

$80 \mathrm{mg}(0.26 \mathrm{mmol}) 14 \mathrm{in} 10 \mathrm{ml}$ acetic acid were reduced as described for 12 and 7: $50 \mathrm{mg}(68 \%) 15, \mathrm{mp}$. $>220^{\circ} \mathrm{C}$ (decomp.) from $\mathrm{CHCl}_{3} / \mathrm{MeOH}$ (9:1 v/v). $-\mathrm{rf}\left(\mathrm{SiO}_{2} ; \mathrm{CH}_{2} \mathrm{Cl}_{2} / \mathrm{MeOH}\right.$ 9:1): 0.45. $-\mathrm{C}_{16} \mathrm{H}_{12} \mathrm{O}_{5}$ (284.3) Calc. C $67.6 \mathrm{H} 4.23$ Found C $67.5 \mathrm{H} 4.44$. - UV $(\mathrm{MeOH}): \lambda \max (\log \varepsilon)=355$ (3.85), 289 (3.98), 258 (4.07), $209 \mathrm{~nm}$ (4.20). - IR: 1695 (COOH), 1623 $\mathrm{cm}^{-1}(\mathrm{C}=\mathrm{O} \cdots \mathrm{HO}) .-{ }^{1} \mathrm{H}-\mathrm{NMR}\left(\left[\mathrm{D}_{6}\right] \mathrm{DMSO}\right): \delta(\mathrm{ppm})=12.62(\mathrm{~s} ; 1 \mathrm{H}$, $\mathrm{OH}), 12.02(\mathrm{~s} ; 1 \mathrm{H}, \mathrm{OH}), 7.72-7.51(\mathrm{~m} ; 2 \mathrm{H}$ aromat. $), 7.10-6.82(\mathrm{~m} ; 3 \mathrm{H}$ aromat.), $4.45\left(\mathrm{~s} ; 2 \mathrm{H}, \mathrm{CH}_{2}\right), 3.60\left(\mathrm{~s} ; 2 \mathrm{H}, \mathrm{CH}_{2}-\mathrm{CO}\right)$. - NI-FAB-MS: $\mathrm{m} / \mathrm{z}$ $=284\left(36 \% ; \mathrm{M}^{-}\right), 283\left[74 ;(\mathrm{M}-\mathrm{H})^{-}\right], 239\left(100 ; 283-\mathrm{CO}_{2}\right)$.

\section{References}

1 A. Retzow, E. Plumier, and W. Wiegrebe, Pharm. Ztg. 126, 2150 (1981).

2 A. Retzow and W. Wiegrebe, Sci. Pharm. 53, 209 (1985).

3 K. Müller, W. Wiegrebe, and M. Younes, Arch. Pharm. (Weinheim) 320,59 (1987) and lit. cited there.

4 J. Martinmaa, J. Juselius, and K. K. Mustakallio, Br. J. Dermatol. 105, Suppl. 20, 52 (1981). 
53 may be the methyl ester of a compound named " $\mathrm{CD} 003$, a 10-succinyl derivative of anthralin", quoted in a publication which contains neither chemical data for comparison nor lit. citations: C. N. Hensby, B. Shroot, A. Chatelus, D. Cavey, J. Allec, and J. Maignan, Agents Actions 21, 247 (1987); ref. C. A. 107, 168452a (1987). - Methoxycarbonyl-propionyl chloride: W. A. Bone, J. J. Sudborough, and C. H. G. Sprankling, J. Chem. Soc. 85, 539 (1904). - G. M. Robinson and R. Robinson, J. Chem. Soc. 127, 180 (1925).
6 K. Krohn, U. Müller, W. Priyono, B. Sarstedt, and A. Stoffregen, Liebigs Ann. Chem. 1984, 306.

7 H. Auterhoff and F. C. Scherff, Arch. Pharm. (Weinheim) 293, 918 (1960).

8 Ch. Marschalk, F. Koenig, and N. Ouroussoff, Bull. Soc. Chim. Fr. 3, 1545 (1936). 
Alle Beiträge in dieser Zeitschrift beginnen auf einer rechten Seite und nicht wie bisher im Anschluß an den vorangehenden Artikel. Es kann deshalb vorkommen, daß eine Seite teilweise oder ganz frei bleibt. Damit entsteht zwar ein etwas größerer Papierbedarf, aber die Publikationsvorbereitungen und die Herstellung der Sonderdrukke werden einfacher, so daß sich insgesamt keine zusätzlichen Kosten ergeben. Auch verlängern sich die Publikationsfristen nicht, da der Jahresumfang der Zeitschrift um die unbedruckt gebliebenen Seiten wächst.

All papers in this issue start on a new right-hand page, instead of immediately following the preceding article. Thus it may happen that part of an article's last page or even a whole (left-hand) page is blank. However, this does not result in higher costs or shorten the issue's contents. Rather, it allows faster publication, speeds up the production of reprints, and helps cutting cost increases. 